\title{
Aplicación del principio de Fermat a la tomografía sísmica. Parte I: fundamentos físicos
}

\author{
Application of Fermat's principle for seismic tomography. \\ Part I: physical foundations
}

\author{
Sebastián Araujo \\ Centro de Investigación en Modelamietno Ambiental - CIMA, Universidad Politécnica Salesiana, Campus Sur, \\ Quito, Ecuador \\ * Autor para correspondencia: jaraujo@ups.edu.ec
}

Manuscrito recibido el 30 de octubre de 2010.Aceptado, tras revisión el I 5 de diciembre de 2010.

\begin{abstract}
Resumen
Este artículo busca analizar la naturaleza de los sismos deduciendo la ecuación fundamental de la tomografía sísmica. Para llegar a ella se analiza la importancia de construir la física desde principios fundamentales, específicamente desde el principio de mínima acción; éste puede ser aplicado a los rayos sísmicos, llegando así al principio de Fermat. Utilizando este principio, originalmente enunciado para la óptica, se obtiene la ecuación fundamental de la tomografía sísmica. Se analizan además las implicaciones de la ecuación de la tomografía para la resolución del problema directo y el problema inverso. Todo este aparataje teórico es imprescindible para obtener imágenes sísmicas de la región de Pisayambo.
\end{abstract}

Palabras clave: Principio de Fermat, tomografía sísmica, principios físicos.

\begin{abstract}
This article has the purpose of analyzing the nature of earthquakes, deducing the fundamental equation of seismic tomography. To get there, we must analyze the importance of building Physics from some fundamental principles, specifically from the principle of least action. This can be applied to seismic rays thus leading to the principle of Fermat, by using this principle, originally enunciated for optics, we can get the fundamental equation of seismic tomography. We must also analyze the implications of the equation of the tomography for the resolution of the direct problem and the inverse one. All this quantity of theory is essential to get seismic images of Pisayambo region.
\end{abstract}

Keywords: Fermat principle, seismic tomography, physics principles. 


\section{Introducción}

La tomografía sísmica se basa en la aplicación de ciertos principios físicos fundamentales que permiten estudiar la propagación de ondas a través de un medio extremadamente heterogéneo como es el caso de la corteza terrestre. El proceso que provoca la onda sísmica que nosotros sentimos como un temblor o terremoto es muy complejo y en la actualidad todavía no se conocen todos sus detalles. Sabemos bien que los terremotos son originados por el movimiento de las placas tectónicas (Sarria Molina, 2007), aunque el mecanismo exacto de la generación de los sismos tectónicos continúa siendo objeto de debate (Koyama, 1997).

Una vez que la onda sísmica se ha generado y ha empezado a propagarse su estudio se simplifica lo cual nos permite, a partir de su camino recorrido, obtener información de la estructura del medio por el cual transita. Esto es lo que básicamente pretende la tomografía sísmica.

Dado que en el Ecuador existe una zona de gran actividad sísmica: el cluster de Pisayambo (Araujo et al., 2009), el interés práctico estará dirigido posteriormente a la aplicación de un programa software de tomografía sísmica a los sismos producidos en Pisayambo (Figura I).

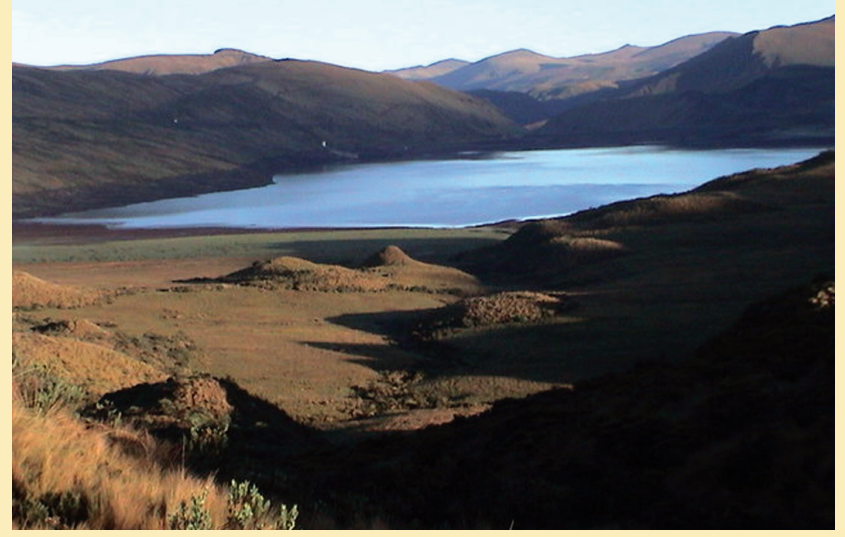

Figura 1. Laguna de Pisayambo que refiere el sitio geográfico donde se localiza el cluster sísmico del mismo nombre.

\section{Principios físicos}

Para estudiar la propagación de una onda sísmica se pueden tomar dos vías. El camino difícil es estudiar todas las ecuaciones para la elastodinámica de los medios continuos (Nolet, 1978). Pero para llegar a la ecuación a partir de la cual podemos desarrollar el es- tudio tomográfico se tiene una alternativa más simple, utilizando un principio físico: el principio de Fermat (Nolet, 1978). La importancia de comenzar la física desde unos principios fundamentales es que siempre ha llevado a una mejor compresión de los fenómenos y por ende hacía una mayor posibilidad de contrastación experimental. Cabe indicar que la vía contraria, partir de axiomas matemáticos sin conexión con la realidad, permite obtener resultados similares mediante el formalismo estético de la herramienta matemática; pero oscurece el discurso y lleva irremediablemente a teorizaciones lejanas de la realidad objetiva (Bunge, 2002).

El principio del tiempo mínimo fue enunciado por Pierre Fermat en 1650 para los rayos luminosos y, en términos generales dice: de todos los caminos que puede seguir la luz para ir de un punto a otro, la luz siempre escoge el camino que requiere el tiempo más corto (Feynman, 197I). El principio del tiempo mínimo es un principio variacional (Elsgoltz, 1983), se trata de una especialización del principio de mínima acción para las ondas. Un principio variacional expresa matemáticamente el hecho de que un fenómeno en la naturaleza ocurre de la forma más óptima posible. Lo interesante de los principios variacionales es que son el punto de partida para poder construir nuestra comprensión de la física contemporánea (Nottale, 1993).

\section{El principio de Fermat}

Para una compresión profunda de lo que significa este principio seguiremos a Landau (Landau y Lifschitz, 198I): una onda sea ésta electromagnética o sísmica se llama onda plana si su amplitud y su dirección de propagación permanecen constantes. Esto no es necesariamente cierto para todas las ondas, pero siempre se pueden definir pequeñas regiones del espacio donde esto se cumpla. Así, se puede delimitar una superficie de la onda donde todos los puntos tengan la misma fase para un instante dado. Si ahora se traza la dirección perpendicular a la superficie de la onda se define una dirección de propagación de la misma. Finalmente, se puede unir todos estos puntos de dirección y obtener lo que se conoce como un rayo. El análisis que sigue es exclusivo para los rayos de naturaleza electromagnética o, en el caso preciso de nuestro interés, rayos sísmicos.

El principio de mínima acción en la mecánica se escribe como:

$$
\delta \int L d t=0
$$


L es el lagrangiano de la partícula. La cuestión es si se puede obtener una expresión similar para los rayos sísmicos. La respuesta inicial es que no, pues, el lagrangiano se calcula:

$$
L=\vec{p} \cdot \frac{\partial \mathcal{H}}{\partial \vec{p}}-\mathcal{H}
$$

Donde $H$ es el hamiltoniano. Para un rayo el hamiltoniano se reemplaza por la frecuencia $\varpi$ y la cantidad de movimiento $\vec{p}$ por el vector de onda $\vec{k}$ :

$$
L=\vec{k} \cdot \frac{\partial \omega}{\partial \vec{k}}-\omega
$$

Si tomamos en cuenta que $\varpi=c k$ :

$$
L=\vec{k} \cdot \vec{c}-\omega
$$

De donde, finalmente, se llega a que el lagrangiano para un rayo es igual a cero.

Para solucionar este problema se puede escribir el principio de mínima acción en forma del principio de Maupertius para la acción $S$ de un sistema con energía constante:

$$
\delta S=\delta \int \vec{p} \cdot d \vec{l}=0
$$

La integral se realiza a través de toda la trayectoria $d \vec{l}$ de la partícula.

Para un rayo, la acción $S$ se corresponde con una función de onda $\psi$ :

$$
\delta \Psi=\delta \int \vec{k} \cdot d \vec{l}=0
$$

La ecuación(6) se conoce como el principio de Fermat.

\section{Aplicación a los rayos sísmicos}

Para poder aplicar el principio de Fermat a las ondas sísmicas es necesario saber si éstas pueden ser consideradas como ondas planas. La solución completa para la ecuación de una onda sísmica contiene muchos términos que no necesariamente son en su conjunto ondas planas. Esto se debe a que la energía sísmica es radiada desde fuentes puntuales, por lo tanto los frentes de onda son siempre esferas en la región más próxima al hipocentro del evento. Sin embargo, cuando la distancia es suficientemente lejana de la fuente, el frente de onda deviene plano. Más aún cuando las soluciones para este caso son ondas planas monocromáticas (Shearer, 2009). Un rayo sísmico entonces puede propagarse entonces desde el hipocentro siendo registrado en la superficie terrestre por una estación sísmica como se esquematiza en la (Figura 2):

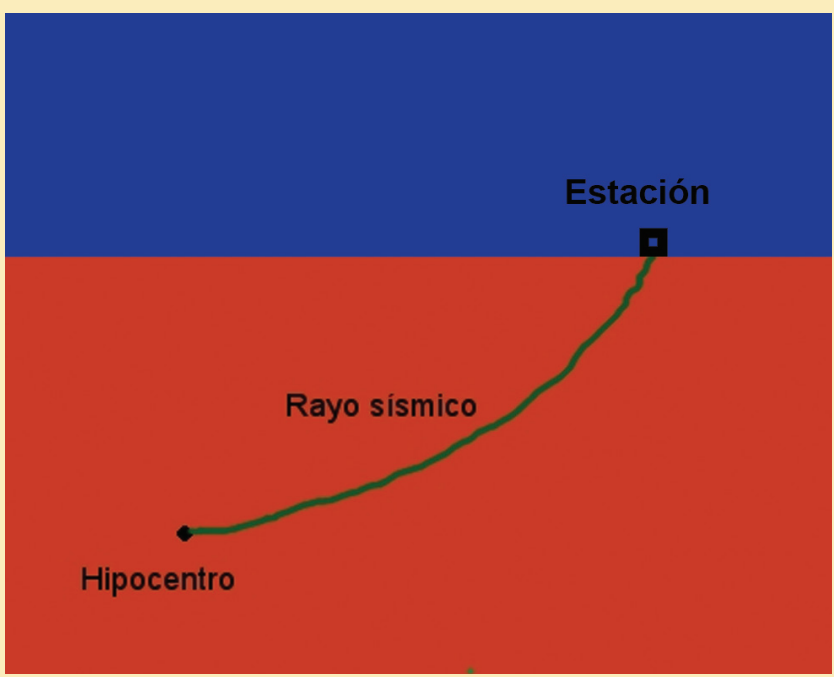

Figura 2. Un sismo que se genera en el hipocentro genera una onda sísmica que si se detecta a suficiente distancia puede ser considerada como una onda plana. La trayectoria de dicha onda entre el hipocentro y la estación se considera como un rayo sísmico.

Se puede así pasar a utilizar (6) y considerar un rayo de frecuencia constante que sigue un camino s:

$$
\delta \int_{s} \frac{\omega}{c(\vec{r})} d s=0
$$

entonces:

$$
\delta \int_{S} \frac{1}{c(\vec{r})} d s=0
$$

La ecuación (8) es el principio de Fermat para los rayos sísmicos. 
Formalmente se puede calcular la variación expresada en (8). Puesto que se trata de una funcional:

$$
F(c(\vec{r}))=\frac{1}{c(\vec{r})}
$$

de una sola variable. Tenemos (Landau y Lifschitz, 1978):

$$
\int_{s}\left(\frac{\partial F}{\partial c} \delta c\right) d s=0
$$

realizando la derivación:

$$
\begin{gathered}
\int_{s}\left(\frac{\partial\left(\frac{1}{c}\right)}{\partial c} \delta c\right) d s=0 \\
\int_{S}-\frac{\delta c}{c^{2}} d s=0
\end{gathered}
$$

La ecuación (12) expresa el hecho de que una variación infinitesimal en la velocidad de propagación del rayo deja invariante el camino de propagación del mismo. O como recíproco, si se consideran dos rayos infinitamente próximos sus velocidades de propagación seguirán siendo las mismas.

Es de interés mostrar que al mismo resultado de (I2) se puede arribar con ciertas aproximaciones que evitan el uso del cálculo variacional. Para ello se perturbará infinitesimalmente la velocidad de propagación del rayo y se aproximará la variación de (8) por:

$$
\int_{s} \frac{1}{c(\vec{r})+\delta c(\vec{r})} d s-\int_{s \Omega} \frac{1}{c(\vec{r})} d s=0
$$

Si se acepta el principio de Fermat, los rayos $S$ y $S_{0}$ son iguales:

$$
\begin{array}{r}
\int_{s}\left(\frac{1}{c(\vec{r})+\delta c(\vec{r})}-\frac{1}{c(\vec{r})}\right) d s=0 \\
\int_{s}\left(\frac{c-c-\delta c}{(c+\delta c) c}\right) d s=0
\end{array}
$$

Si se considera la aproximación $c+\delta c \sim c$ otra vez se llega a la expresión (12).

Ahora se requiere considerar el tiempo de propagación de un rayo sísmico. Este dato es de interés pues es la magnitud que miden los sismómetros. Una estación sísmica medirá un tiempo de propagación del rayo, que se puede entender como una integral de línea del espacio recorrido dividido para la velocidad:

$$
t=\int_{s} \frac{d s}{c(\vec{r})}
$$

Por otro lado, se puede haber calculado el tiempo de propagación del sismo utilizando un modelo:

$$
t_{\mathrm{o}}=\int_{s_{\mathrm{O}}} \frac{d s}{c_{\mathrm{o}}(\vec{r})}
$$

De la forma de las ecuaciones (I4) y (I5) se puede observar que el principio de Fermat (8) es un variacional sobre los tiempos de propagación de los rayos.

A la diferencia entre el tiempo medido y el tiempo calculado la llamamos residuo:

$$
r=t-t_{0}
$$

Ahora viene la aproximación clave que permite llegar a la tomografía sísmica (Nolet, 1978); se supondrá que el error que se comete al calcular el tiempo de propagación es un infinitésimo respecto al tiempo medido. Esto quiere decir que $r$ tiende en límite al diferencial de tiempo. Con ello podemos igualar el principio variacional (8) al residuo:

$$
\delta t=\delta \int_{s} \frac{1}{c(\vec{r})} d s
$$

Si se reemplaza la variación obtenida en (12) llegamos a:

$$
\delta t=\int_{s}-\frac{\delta c}{c^{2}} d s
$$

La ecuación (18) es el fundamento de la tomografía sísmica. También se puede obtener fácilmente (18) si se observa que: 


$$
t-t_{\mathrm{o}}=\int_{S} \frac{1}{c(\vec{r})} d s-\int_{s o} \frac{1}{c_{o}(\vec{r})} d s
$$

Las velocidades de aproximación difieren en un infinitésimo $c=c_{o}+\delta c$. De ahí podemos retomar la ecuación (13) y arribar nuevamente a (I8).

Para terminar, se escribirá la ecuación fundamental de la tomografía sísmica en forma diferencial. Para ello simplemente se toma la derivada parcial del tiempo de propagación del rayo respecto a la velocidad:

$$
\frac{\partial t}{\partial c}=\frac{\partial}{\partial c} \int \frac{d s}{c}
$$

Se pueden intercambiar los operadores derivada en integral:

$$
\frac{\partial t}{\partial c}=\int\left(\frac{\partial \frac{1}{c}}{\partial c}\right) d s
$$

Si se efectúa la derivada se llega al valor de la integral:

$$
\int \frac{d s}{c^{2}}=-\frac{\partial t}{\partial c}
$$

Si se reemplaza en (18) finalmente obtenemos:

$$
\delta t=\frac{\partial t}{\partial c} \delta c
$$

De la ecuación (23) podemos partir para cualquier proceso de tomografía sísmica (Monteiller et al., 198I).

\section{La tomografía sísmica}

La ecuación (23) permite resolver dos tipos de problemas. El primero consiste en calcular el tiempo de propagación conocida la velocidad del medio. El resolver el problema directo implica a su vez trazar el rayo sísmico desde la fuente hasta el receptor.Aquí el principio de Fermat es fundamental pues nos permite calcular el tiempo de propagación del rayo mismo si no conocemos exactamente la verdadera trayectoria del rayo sísmico pues, en primera aproximación, el tiempo de propagación es insensible a las perturbaciones en el camino del rayo (Shearer, 2009). Es por el principio de Fermat entonces que podemos, si los eventos se producen en profundidad y tenemos estaciones en la superficie, aproximar los rayos por líneas rectas como se muestra en la figura 3 para eventos localizados por el Observatorio de Estrasburgo en Francia (http:// renass.u-strasbg.fr/).

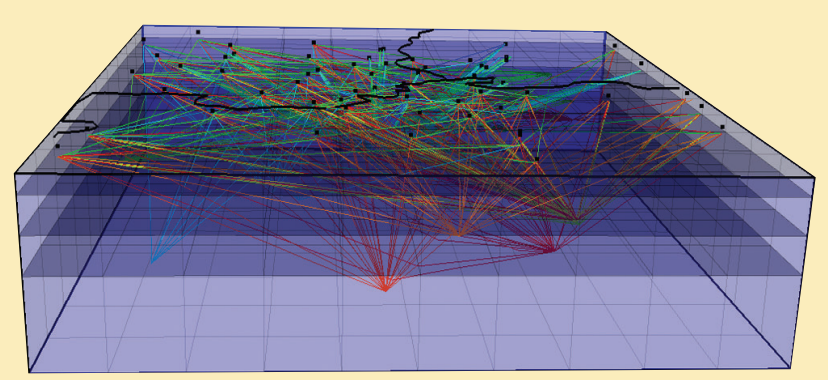

Figura 3. Rayos sísmicos rectilíneos trazados desde el hipocentro hasta las estaciones receptoras en la superficie. El que podamos considerar a los rayos sísmicos como líneas rectas es una consecuencia directa del principio de Fermat. Imagen http://renass.u-strasbg.fr/ ray $2 \mathrm{mesh} / \mathrm{img} / 10$. png.

El segundo problema es más complicado pues, se trata de calcular la velocidad del medio conocidos los tiempos de propagación, es el problema inverso. Este ejercicio se complica ya que en la integral (I8) el mismo camino de integración $s(c)$ es función de la velocidad. Para el problema inverso, por tanto los errores ya no serán de primer orden.

La forma aceptada de atacar el problema inverso consiste en tener no sólo un rayo sino $i$ eventos registrados en $k$ estaciones. Entonces:

$$
t_{o b a_{i}-} t_{i}^{k}=\frac{\partial t_{i}^{k}}{\partial m} \delta m_{i}
$$

Los residuos, las diferencias, entre los tiempos observados y los tiempos calculados se relacionan con la perturbación modelo de velocidades $\delta m_{i}$ :

$$
r_{i}^{k}=\frac{\partial t_{i}^{k}}{\partial m} \delta m_{i}
$$

Podemos escribir (25) como matrices (Monteiller et al., 2005):

$$
r=G \delta m
$$

Encontrar el modelo de velocidades $m$ de un medio conocidos los tiempos de propagación de los rayos símicos es lo que se conoce como tomografía 
sísmica. Para resolver este problema inverso no lineal y subdeterminado se deben hacer uso de aproximaciones probabilísticas (Araujo, 2008).

\section{Conclusiones}

Definir la física desde unos principios físicos fundamentales es una tarea que siempre permite la creación de nueva ciencia. En el caso de la sismología, una vez definido el concepto de rayo sísmico se puede hacer un alcance del principio de mínima acción para estos rayos. Este principio se conoce como principio de Fermat y su aplicación lleva a la ecuación de la cual podemos partir para obtener imágenes tomográficas del interior de la Tierra.

Dadas las dificultades matemáticas que se presentan al tratar de resolver tanto el problema directo como el problema inverso, la aproximación de los rayos sísmicos rectilíneos pueden ser un interesante punto de partida para llegar a una tomografía de la región de Pisayambo (Tarantola, 2007).

\section{Bibliografía}

Araujo S., L. Troncoso y M. Ruiz. 2009. Relocalización por dobles diferencias del clúster sísimico de Pisayambo. La Granja. 10 (2). Pp. 27-34 ISSN: I 390-3799

Araujo, S. 2008. Solución probabilística del problema inverso. Memorias del XI Encuentro de Matemáticas y sus aplicaciones. Escuela Politécnica Nacional.

Bunge, M. 2002. Ser, Saber, Hacer. Paidós.

Elsgoltz. 1983. Ecuaciones diferenciales y cálculo variacional. Mir.
Feynman, R. 197I. Física Tomo I: Mecánica, Radiación y Calor. Fondo Educativo lberoamericano.

Koyama, J. 1997. The complex faulting process of earthquakes. Kluwer.

Landau L.D., E.M. Lifschitz. I978. Mecánica. Editorial Reverté.

Landau L.D., E.M. Lifschitz. 198I. Teoría Clásica de los Campos. Editorial Reverté.

Monteiller,V., JL. Got, J.Virieux y P. Okubo. 2005. An eficient algorithm for Doubble diference tomography and location in heterogeneous media with application to the Kilauea volcano.J. Geophys. Res.I I0. BI 2306.

Nolet, G. 2008. A Breviary Of Seismic Tomography - Imaging The Interior Of The Earth And Sun. Cambridge: University Press.

Nolet, G. 1987. Seismic tomography: with applications in global seismology and exploration geophysics. Springer.

Nottale, L. 1993. Fractal space-time and microphysics: towards a theory of scale relativity. World Scientific.

Sarria Molina, A. 2007. Dinámica Terrestre: visión integral para la ingeniería moderna. Universidad de los Andes.

Shearer, P. 2009. Introduction to seismology. Cambridge: University Press.

Taranto,A.2007.Inverse Problems:Exercices, With mathematica, matlab, and scilab solutions. Submited to SIAM. 